\title{
Tumour Necrosis Factor Alpha and Oxidative Stress in the Breath Condensate of Those with Non-Small Cell Lung Cancer
}

\author{
Enoch Chan ${ }^{1}$, Thevaki Sivagnanam ${ }^{2}$, Qi Zhang ${ }^{1}$, Craig R. Lewis ${ }^{1,3}$, Paul S. Thomas ${ }^{1,2 *}$ \\ ${ }^{1}$ Inflammation and Infection Research Centre and Prince of Wales Clinical School, Faculty of Medicine, University of New South \\ Wales, Sydney, Australia; ${ }^{2}$ Department of Respiratory Medicine, Prince of Wales Hospital, Randwick, Australia; ${ }^{3}$ Department of \\ Medical Oncology, Prince of Wales Hospital, Randwick, Australia. \\ Email: *paul.thomas@unsw.edu.au
}

Received July $31^{\text {st }}, 2012$; revised August $30^{\text {th }}, 2012$; accepted September $15^{\text {th }}, 2012$

\begin{abstract}
Background and Aims: Lung cancer is a leading cause of cancer mortality worldwide and is associated with the release of tumour necrosis factor- $\alpha$ (TNF- $\alpha$ ), subsequent cellular apoptosis and the generation of oxidative stress. Exhaled breath condensate (EBC) analysis is a non-invasive method for sampling biofluids from the lower respiratory tract. This study aimed to evaluate possible biomarkers of lung cancer by measuring the levels of TNF- $\alpha$ and the oxidation of ascorbic acid in EBC. Patients with lung cancer were enrolled into the study prior to treatment, during treatment and post-treatment, and results compared with an age-matched control population. Material and Methods: Patients with Stages II-IV non small cell lung cancer (NSCLC) were recruited prior to and at stages of their treatment. EBC levels of TNF- $\alpha$, and rate of ascorbic acid oxidation were measured. Results: A total of 19 patients with NSCLC (mean age $71.37 \pm 7.77 \mathrm{yr}$ ) and 25 age-matched control subjects were enrolled. Levels of EBC TNF- $\alpha$ were elevated in the EBC of patients with lung cancer compared with control subjects $(1.02 \pm 0.07 \mathrm{pg} / \mathrm{ml}$ vs $0.51 \pm 0.06 \mathrm{pg} / \mathrm{ml}, \mathrm{p}<0.0001)$. Moreover, the rate of ascorbic acid oxidation was significantly greater in the $\mathrm{EBC}$ of patients with lung cancer compared with control subjects $(2.20 \%$ [0.4 - 11.0] vs $1.00 \%$ [0.1 - 8.5], $\mathrm{p}=0.0244)$. Conclusion: TNF- $\alpha$ and the rate of ascorbic acid oxidation was elevated in the EBC of patients with lung cancer regardless of treatment. Longitudinal studies in a larger population are required to evaluate these markers for the effect of treatment and prognosis.
\end{abstract}

Keywords: Exhaled Breath Condensate; Lung Cancer; Ascorbic Acid Oxidation; Tumour Necrosis Factor Alpha

\section{Introduction}

Lung cancer is a leading cause of cancer mortality worldwide, with over 1.6 million new cases and over 1.3 million deaths each year [1-3]. It contributes to 157,000 deaths and $28 \%$ of cancer deaths annually in the USA, and $19 \%$ of cancer deaths in Australia [4-5]. Despite advances in treatment strategies, long-term mortality has not improved significantly in the last 20 years, with the overall 5 -year survival being $16 \%$ in the USA. This is mainly due to the late onset of symptoms resulting in patients who present with advanced and incurable disease. Such symptoms often relate to disseminated disease such as bony metastases or to locally advanced disease with breathlessness, lung infection or haemoptysis. However, when lung cancer is discovered earlier, radical treatment and potentially curative surgery may improve 5 -year survival rates (from $20 \%$ in those with Stage III lung cancer

${ }^{*}$ Corresponding author. to $70 \%$ in patients with Stage I disease [6-8]. Current methods of diagnosing lung disease such as CT guided tissue biopsy or bronchoscopic biopsy and bronchialveolar lavage are associated with invasive risks $[9,10]$. Therefore a simple, non-invasive and relatively inexpensive method of screening for lung cancer in at-risk populations may potentially detect early disease and reduce lung cancer mortality.

Recent advances in tumour biology and angiogenesis has promoted studies into potential tumour biomarkers [11-13]. Traditionally, blood or tumour samples are used to identify biomarkers but analysis of exhaled breath condensate (EBC) provides a promising, non-invasive approach that could allow screening of smokers for the diagnosis of lung cancer. EBC analysis is the collection of epithelial lining fluid from the lower respiratory tract via condensation of exhaled breath. EBC consists primarily of water vapour as well as various aerosolised particles including non-volatile organic compounds and 
volatile organic compounds (VOCs) that may be used to identify lung pathology [11-13].

Lung cancer biomarkers and those associated with tumour lysis may be able to aid in screening of lung cancer and possibly predict a patient's response to cancer treatment [14]. TNF- $\alpha$ is highly expressed in tumours and is elevated in the serum of patients with non-small cell lung cancer (NSCLC) when compared with control subjects $[15,16]$. TNF- $\alpha$ is elevated in the EBC of patients with lung cancer when compared with control subjects [16]. TNF- $\alpha$ is also a mediator for initiating cellular apoptosis but its apoptotic pathways may be circumvented by certain tumour cell characteristics [17]. Therefore levels of TNF- $\alpha$ may be raised in the EBC of patients with lung cancer and further increased in treatment-responsive tumours. Cigarette smoking and environmental pollutants can release TNF- $\alpha$ from alveolar macrophages which in turn generate oxidative stress in the lung. In addition, there is an increase in the levels of known markers of oxidation in the EBC derived from smokers, such as hydrogen peroxide, 8-isoprostane, as well as those of the nitrosative pathway e.g. nitrate, and S-nitrosothiols [1821]. Oxidative stress is also associated with the induction of lung cancer, possibly by indirect and direct mutagenesis (e.g. DNA adduct formation, DNA double strand breaks, DNA mutation) [22]. Furthermore, there is increased oxidative stress in cancer cells compared with normal cells $[23,24]$. This may be due to oncogenic signals that increase production of reactive oxygen species (ROS) $[25,26]$ or the increased metabolic demand of rapidly growing and differentiating cancer cells [27].

In the presence of oxygen, ROS including hydroxyl radicals, superoxides and organic radicals generate other downstream ROS including hydrogen peroxide and organic hydroperoxides. These highly reactive radicals oxidise anti-oxidants including ascorbic acid. Both radiotherapy and chemotherapy induce ROS at the site of the tumour to damage cell lipid membranes and the DNA of malignant cells, thus capturing this phenomenon for therapeutic gain. The reduced ability of tumour cells to repair damaged DNA increases their vulnerability to chemoand radiotherapy when compared with normal, healthy cells $[28,29]$.

It was postulated that increased ROS as an indication of elevated oxidative stress could be measured in the EBC of patients with NSCLC and undergoing treatment. ROS activity in EBC may be assessed by the ex-vivo oxidation of ascorbic acid. The ascorbate reacts with the oxidative species and is degraded, thus its rate of degradation can be analysed and compared between patients pre- and post-therapy and with control subjects.

\section{Materials and Methods}

\subsection{Subject Recruitment}

This study was approved by the South Eastern Sydney Area Health Service Research Ethics Committee. Participants with NSCLC were recruited from the Prince of Wales Hospital, NSW. Studied subjects were divided into a pre-treatment group, a group undergoing treatment and a post-treatment group. The inclusion/criterion for the pre-treatment group was that the subject had to be eligible for chemotherapy, radiotherapy or surgery. The exclusion criterion was that they must not have had any chemotherapy, radiotherapy or surgery for their lung cancer before EBC sample collection. Treatment definetion for $\mathrm{EBC}$ collection in subjects during treatment and post-treatment was defined as chemotherapy and/or radiotherapy, \pm surgery. EBC for the post-treatment group was collected at least three weeks after the final treatment. Control subjects without lung cancer were recruited from the orthopaedic ward and were age and gender matched. The exclusion criterion for control subjects was a past or current history of lung cancer.

Informed consent was obtained and a questionnaire completed for demographic details and relevant medical details including information on smoking history, (exsmokers defined as not having smoked for at least one year), medical history, medication, and staging and histological typing of lung cancer.

\subsection{EBC Collection}

EBC was collected as previously described [11]. Briefly, prior to collecting $\mathrm{EBC}$, the subjects were instructed to rinse their mouths with water. EBC was then collected for $15 \mathrm{~min}$ with tidal breathing into a unidirectional mouthpiece connected to a custom unsiliconised glass collection device and condensed using wet ice at approximately $4^{\circ} \mathrm{C}$. Excess saliva was swallowed.

The EBC was aliquoted into $120 \mu \mathrm{l}$ samples in $1.5 \mathrm{ml}$ Lobind Eppendorf tubes (POCD Scientific, Artarmon, NSW) and degassed at $0.5 \mathrm{~L} / \mathrm{min}$ with high purity argon gas (BOC, Sydney) for one minute. All samples were immediately stored at $-80^{\circ} \mathrm{C}$ for subsequent analysis.

\subsection{Measurements of Tumour Necrosis Factor- $\alpha$}

TNF- $\alpha$ levels were measured using a commercial sandwich enzyme-linked immunosorbent assay (ELISA) (Invitrogen, Mulgrave, Victoria), with a sensitivity of $<0.09$ $\mathrm{pg} / \mathrm{ml}$. EBC samples were assayed in duplicates. The range for this assay was $0-32 \mathrm{pg} / \mathrm{ml}$ and the limit of detection was $0.2 \mathrm{pg} / \mathrm{ml}$. 


\subsection{Ascorbic Acid Oxidation}

Ascorbic acid oxidation was measured by analysing the rate of oxidation of ascorbic acid by ROS in EBC using the reaction: Ascorbic Acid $+\mathrm{RO} \bullet \rightarrow$ Ascorbyl radical + ROH. [30] Briefly, an aliquot of $20 \mu \mathrm{l}$ of $1.65 \mathrm{M}$ ascorbic acid solution (Sigma Aldrich, Australia) was added to $200 \mu \mathrm{l}$ of de-ionised water and $100 \mu \mathrm{l}$ of EBC in a black quartz cuvette. The rate of ascorbic acid oxidation as determined by change in absorbance at $265 \mathrm{~nm}$ was monitored at 30 second intervals for 210 seconds. At the end of this period and to determine the presence of any transition metals, $10 \mu \mathrm{l}$ of $660 \mu \mathrm{M}$ ethylene diamine tetra acetic acid solution was added as a chelator and the rate of oxidation was monitored at 30 second intervals for a further 150 seconds.

\subsection{Statistical Analysis}

Statistical analyses were performed using Graph Pad Prism 5 (Graph Pad, La Jolla CA). TNF- $\alpha$ data were normally distributed and analysed by parametric statistics (one-way ANOVA, Bonferroni post-hoc test and independent unpaired 2-tailed t-test where appropriate). For follow-up patients Repeated Measures ANOVA was used. Data for parametric statistics are presented as mean \pm S.E.M. Ascorbic acid oxidation did not follow a Gaussian distribution and non-parametric tests were used; Kruskal-Wallis test for data with multiple groups and Mann-Whitney U test for data with two groups. Data for non-parametric statistics are presented as median [range].

\section{Results}

Of the 44 individuals that were enrolled into the study, 19 had been diagnosed with NSCLC (11 male, 8 female). Control subjects comprised 11 healthy smokers/ex-smokers (6 males, 5 females), and 14 healthy non-smokers ( 5 males, 9 females). Subjects gave variable volumes of EBC and as a result, some patients had inadequate samples collected for the analysis of every marker. Subject demographics are summarised in Table 1. All patients with lung cancer undergoing treatment or who were posttreatment had a positive response to treatment with reduction in tumour size. Demographic characteristics are summarised in Table 2.

\subsection{Tumour Necrosis Factor- $\alpha$}

Patients with lung cancer had significantly higher concentrations of TNF- $\alpha$ than control subjects $(-0.08 \pm 0.03$ $\mathrm{pg} / \mathrm{ml}$ vs $-0.42 \pm 0.05 \mathrm{pg} / \mathrm{ml}, \mathrm{p}<0.0001$; Figure 1). There was no significant difference in TNF- $\alpha$ concentration between pre-treatment, treatment and post treatment groups. However, each treatment group was significantly
Table 1. Subject demographics.

\begin{tabular}{ccccc}
\hline & \multicolumn{3}{c}{ Control Subjects } & $\begin{array}{c}\text { Subjects } \\
\text { with Lung } \\
\text { Cancer }\end{array}$ \\
\cline { 2 - 5 } & Non-smokers & $\begin{array}{c}\text { Smokers/Former } \\
\text { smokers }\end{array}$ & Total & 19 \\
\hline $\mathrm{N}$ & 14 & 11 & 25 & 19 \\
$\begin{array}{c}\text { Age } \\
(\text { mean } \pm \text { SD) } \\
\text { Male/Female }\end{array}$ & $73.6 \pm 11.5$ & $75.4 \pm 8.7$ & $74.4 \pm 10.2$ & $71.4 \pm 7.8$ \\
\hline
\end{tabular}

Table 2. Demographic characteristics for subjects with lung cancer.

\begin{tabular}{lc}
\hline $\mathrm{N}$ & 19 \\
Age (mean $\pm \mathrm{SD})$ & $71.4 \pm 7.8$ \\
Male/Female & $11 / 8$ \\
Smoking (current/former/never) & $3 / 12 / 4$ \\
Adenocarcinoma & 8 \\
Large cell carcinoma & 3 \\
Squamous cell carcinoma & 5 \\
NSCLC (NOS) & 3 \\
Stage I/II/III/IV & $1 / 3 / 6 / 9$ \\
Treatment pre/during/post & $8 / 7 / 10$ \\
Follow-up during/post & $3 / 3$ \\
\hline
\end{tabular}

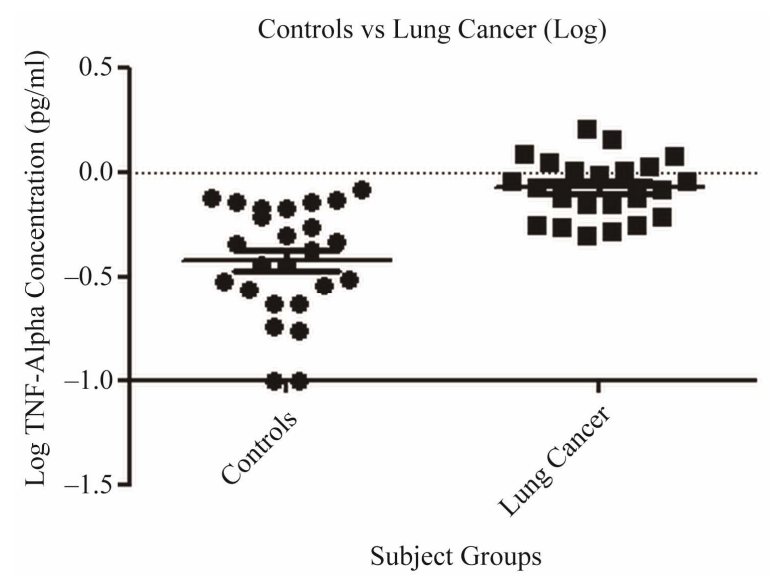

Figure 1. EBC TNF- $\alpha$ levels in control subjects $(n=25)$ versus subjects with lung cancer $(n=23)$ in EBC $(1.02 \pm$ $0.07 \mathrm{pg} / \mathrm{ml}$ vs $0.51 \pm 0.06 \mathrm{pg} / \mathrm{ml}, \mathrm{p}<\mathbf{0 . 0 0 0 1})$. Note: Two subjects with lung cancer had pretreatment, during treatment and post treatment follow up results included.

different when compared with both non-smoker control subjects and control smokers/ex-smokers $(p<0.05$, Figure 2). Treatment did not significantly affect the level of EBC TNF- $\alpha$ nor were there significant differences observed between the stages of lung cancer (Stage II $(n=6)$ $0.86 \pm 0.12$, Stage III $(\mathrm{n}=6) 1.09 \pm 0.19$, Stage IV $(\mathrm{n}=$ 10) $1.09 \pm 0.08)$ 


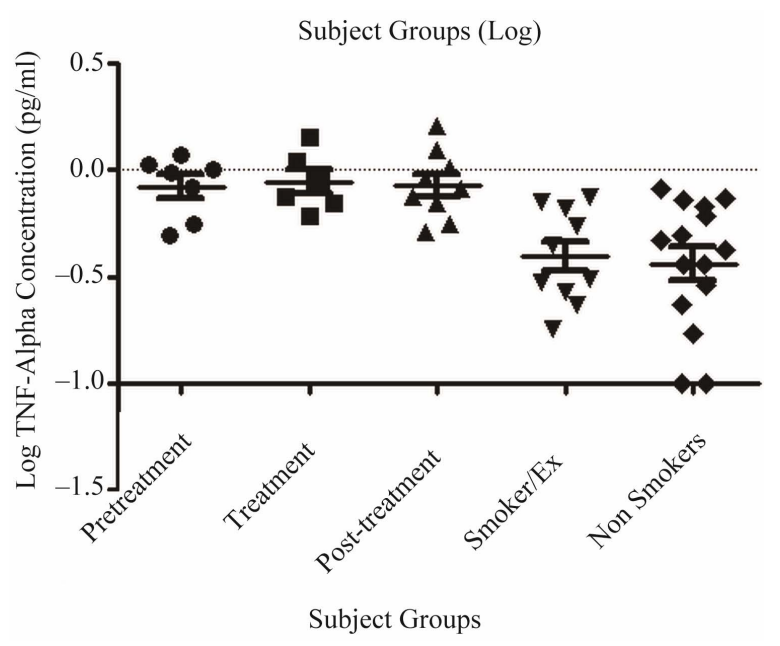

Figure 2. Comparison of EBC TNF- $\alpha$ levels during treatment in comparison with control groups. Significant differences were found between any of the treatment sub-groups to both control subgroups groups $(p<0.05)$. (Means: pretreatment $(n=7) 1.02 \pm 0.11$, treatment $(n=7) 1.00 \pm 0.13$, post-treatment $(\mathrm{n}=9) 1.04 \pm 0.13$, smokers/ex-smokers $(\mathrm{n}=$ 10) $0.54 \pm 0.08$, non-smokers $(\mathrm{n}=15) 0.49 \pm 0.08)$. Note: Two subjects with lung cancer had pretreatment, during treatment and post treatment follow up results included.

\subsection{Ascorbic Acid Oxidation}

Patients with lung cancer had significantly greater percentage degradation of ascorbic acid from baseline than control subjects $(2.20 \%$ [ $0.4-11.0]$ vs $1.00 \%$ [0.1 - 8.5], $\mathrm{p}=0.024$; Figure 3). No significant difference between the rates of ascorbic acid degradation was found between treatment and control subgroups (Figure 4). No significant difference was observed between lung cancer stages.

\section{Discussion}

This study shows a significant increase in the levels of EBC TNF- $\alpha$ and in the oxidation rate of ascorbic acid in the EBC of patients with primary lung cancer when compared with control subjects.

TNF- $\alpha$ can activate the cell-mediated response against neoplastic cells as well as mediate tumourigenesis in lung cancer, such as promoting neoplastic cell growth and inhibiting the anti-tumour immune response [31]. Patients with lung cancer had greater levels of EBC TNF- $\alpha$, independent of whether they were yet to commence treatment, undergoing treatment at the time of collection or had completed their treatment, when compared with healthy controls. This finding is consistent with a previous study that reported statistically elevated levels of TNF- $\alpha$ in the EBC of patients with lung cancer prior to treatment when compared with controls [16].

This study also observed sequential elevation of TNF$\alpha$ levels from samples of EBC from two patients as they

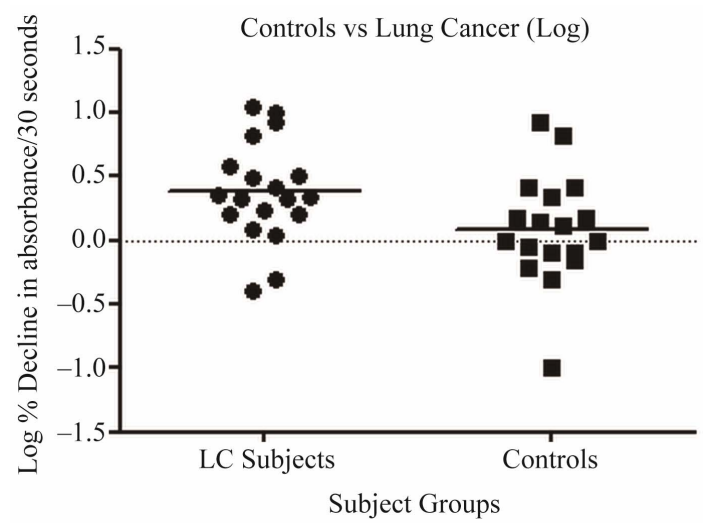

Figure 3. The rate of decline of ascorbic acid in the EBC of patients with lung cancer $(n=19)$ versus control subjects $(n$ $=18) .(2.20 \%[0.4-11.0]$ vs $1.00 \%$ [0.1 -8.5$], p=0.024)$.

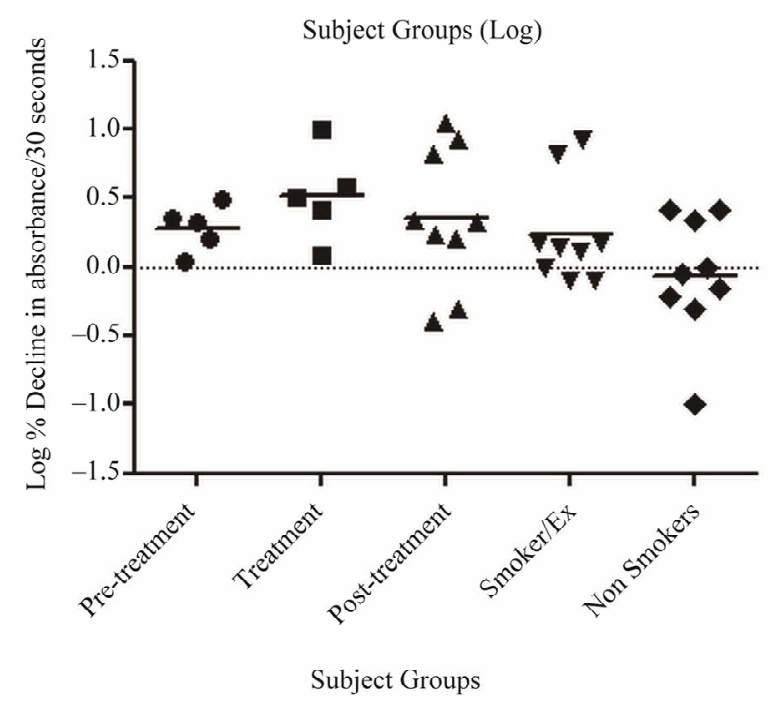

Figure 4. Comparison of the rate of degradation of ascorbic acid in the EBC between different treatment sub-groups and control sub-groups. No differences were found between these subgroups. (Medians: pre-treatment $(n=5)$ 2.04 [1.1 3.1], treatment $(n=5) 4.18$ [1.2 - 10.1], post-treatment $(n=9)$ 3.8 [0.4 - 11.0], smokers/ex-smokers $(\mathrm{n}=9) 2.6$ [0.8 - 8.5], non-smoker $(\mathrm{n}=9) 1.2[0.1$ - 2.6]).

progressed from pre-treatment to treatment and then finally follow up post-treatment, but this trend was not observed over the group (Figure 3). Both patients had positive responses to treatment, which supports the antiproliferative effects of TNF- $\alpha$ in higher concentrations, but this observation would need to be confirmed in larger studies [17]. This is the first study, to our knowledge, that has analysed TNF- $\alpha$ in the EBC of patients before, during and post treatment. The group data did not suggest that there was a clear response in EBC TNF- $\alpha$ levels to treatment.

Compared to normal cells, neoplastic cells are in a pro-oxidative state that leads to intrinsic oxidative stress. 
Cancer cells have higher levels of ROS than normal cells, and ROS are, in turn, responsible for the maintenance of the cancer phenotype [25-27]. Ascorbic acid is oxidised by ROS produced by these neoplastic cells [32]. In this study there was an increased rate of ascorbic acid degradation in the EBC of patients with lung cancer compared with healthy control subjects. This is a novel finding from our study, as there are no previous publications in the literature regarding the increased rate of ascorbic acid oxidation with lung cancer, but it is supported by previous work which showed elevated $\mathrm{H}_{2} \mathrm{O}_{2}$, decreased total anti-oxidants as measured by a total anti-oxidant assay, and decreased $\mathrm{pH}$ [11]. Interestingly, the current data did not suggest a significant difference between the smoking control group and those with NSCLC, suggesting that the induction of ROS by smoking remains a major contributor to EBC ROS. Furthermore, ROS did not significantly differ after the initiation of treatment, which may reflect the small sample size, but indicates that there is not a major change in the ROS. It may be that a different and more sensitive marker may be required to be used for assessing clinical response to treatment [33].

This study conformed to the recommendations provided by the ERS/ATS Task Force however, there were some limitations. [34] It was a single centre, cross-sectional observational study, with a modest sample size, which undoubtedly affects the power of this study.

ROS are unstable in EBC thus rapid freezing of the samples at $-80^{\circ} \mathrm{C}$ is required until they were analysed. Furthermore, samples were thawed and kept on ice during analysis. The ascorbic acid oxidation reaction is catalysed by transitional metals such as copper (II) ions $[35,36]$ and thus the presence of different levels of transitional metals may also affect the rate at which ascorbic acid is oxidised. There was no significant increase in the rate of ascorbic acid oxidation during treatment for lung cancer, or between the treatment subgroups and control subgroups. Despite these limitations, we believe that our study provides further convincing evidence that analysis of exhaled breath is promising, as it shows that the technique is feasible and can be performed in those with significant lung disease.

As previously mentioned, further longitudinal investigations are needed, in large multicentre studies, evaluating levels of biomarkers in patients with lung cancer at various stages through their treatment to further support our findings.

\section{Conclusion}

TNF- $\alpha$ and the rate of ascorbic acid oxidation are increased in the EBC of patients with lung cancer regardless of treatment status. Longitudinal studies would assist in the evaluation of the clinical use of these markers in diagnosing and assessing treatment efficacy in lung cancer.

\section{Acknowledgements}

The authors thank the participants for their time and Beth Ivimey for her kind assistance with both the patients and recruitment.

\section{REFERENCES}

[1] J. Ferlay, H.-R. Shin, F. Bray, et al., "Parkin. Estimates of Worldwide Burden of Cancer in 2008: GLOBOCAN 2008," International Journal of Cancer, Vol. 127, No. 12, 2010, pp. 2893-2917. doi:10.1002/ijc.25516

[2] C. D. Mathers and D. Loncar, "Projections of Global Mortalitly and Burden of Disease from 2002 and 2030," PLoS Medicine, Vol. 3, 2006, p. 3442. doi:10.1371/journal.pmed.0030442

[3] A. Hemal, R. Siegel, E. Ward, et al., "Cancer Statistics 2008," CA-A Cancer Journal for Clinicians, Vol. 58, No. 2, 2008, pp. 71-96. doi:10.3322/CA.2007.0010

[4] (AIHW) AIoHaW, "ACIM (Australian Cancer Incidence and Mortality)," AIHW, Canberra, 2010.

[5] U. Ahmad, M. Ruschel and F. C. Detterbeck, "Lung Cancer: Facts, Figures and Reflections on Spending," Journal of Cancer Therapy, Vol. 3, No. 2, 2012, pp. 123-126. doi: $10.4236 /$ jct.2012.32016

[6] C. Mountain, "Revisions in the International System for Staging Lung Cancer," Chest, Vol. 111, No. 6, 1997, pp. 1710-1717. doi:10.1378/chest.111.6.1710

[7] W. De Weaver, S. Stroobants, J. Coden, et al., "Integrated PET/CT in the Staging of Non Small Cell Lung Cancer: Technical Aspects and Resection for Lung Cancer," European Respiratory Journal, Vol. 33, 2009, pp. 201212. doi: $10.1183 / 09031936.00035108$

[8] A. McWilliams, B. Lam and T. Sutedja, "Early Proximal Lung Cancer Diagnosis and Treatment," European Respiratory Journal, Vol. 33, 2009, pp. 656-665. doi:10.1183/09031936.00124608

[9] P. B. Bach, D. E. Niewoehner and W. C. Black, "Screening for Lung Cancer: The Guidleines," Chest, Vol. 123, No. 1, 2003, pp. 83S-88S. doi:10.1378/chest.123.1_suppl.83S

[10] E. Edell, S. Lam, H. Pass, et al., "Detection and Localisation of Intraepithelial Neoplasia and Invasive Carcinoma Using Fluorescence-Reflectance Bronschospcopy: An International, Multicenter Clinical Trial," Journal of Thoracic Oncology, Vol. 4, 2009, pp. 49-54.

[11] H. P. Chan, V. Tran, C. Lewis and P. S. Thomas, "Elevated Levels of Oxidative Stress Markers in Exhaled Breath Condensate," Journal of Thoracic Oncology, Vol. 4, No. 2, 2009, pp. 172-178. doi:10.1097/JTO.0b013e3181949eb9

[12] H. P. Chan, C. Lewis and P. S. Thomas, "Exhaled Breath Analysis: Novel Approach for Early Detection of Lung Cancer," Lung Cancer, Vol. 63, No. 2, 2009, pp. 164-168. 
doi:10.1016/j.lungcan.2008.05.020

[13] D. H. Conrad, J. Goyette and P. S. Thomas, "Proteomics as a Method for Early Detection of Cancer: A Review of Proteomics, Exhaled Breath Condensate, and Lung Cancer Screening," Journal of General Internal Medicine, Vol. 23, No. 1S, 2007, pp. 78-84.

[14] K. L. Reckamp, B. K. Gardner, R. A. Figlin, et al., "Tumor Response to Combination Celecoxib and Erlotinib Therapy in Non-Small Cell Lung Cancer Is Associated with a Low Baseline Matrix Metalloproteinase-9 and a Decline in Serum-Soluble E-Cadherin," Journal of Thoracic Oncology, Vol. 3, No. 2, 2008, pp. 117-124. doi:10.1097/JTO.0b013e3181622bef

[15] D. Derin, H. O. Soydinc, N. Guney, et al., " Serum Levels of Apoptosis Biomarkers and TNF-Alpha in Nonsmall Cell Lung Cancer," Lung Cancer, Vol. 59, No. 2, 2008, pp. 240-245. doi:10.1016/j.lungcan.2007.08.005

[16] E. Dalaveris, T. Kerenidi, A. Katsabeki-Katsafli, et al., "VEGF, TNF-Alpha and 8-Isoprostane Levels in Exhaled Breath Condensate and Serum of Patients with Lung Cancer," Lung Cancer, Vol. 64, No. 2, 2009, pp. 219-225. doi:10.1016/j.lungcan.2008.08.015

[17] P. W. Szlosarek and F. R. Balkwill, "Tumour Necrosis Factor $\alpha$ : A Potential Target for the Therapy of Solid Tumors," The Lancet Oncology, Vol. 4, No. 1, 2003, pp. 565-573. doi:10.1016/S1470-2045(03)01196-3

[18] P. Montuschi and P. J. Barnes, "Analysis of Exhaled Breath Condensate for Monitoring Airway Inflammation," Trends in Pharmacological Sciences, Vol. 23, No. 5, 2002, pp. 232-237. doi:10.1016/S0165-6147(02)02020-5

[19] K. Garey, M. M. Neuhauser, R. A. Robbins, L. H. Danziger and I. Rubinstein, "Markers of Inflammation in Exhaled Breath Condensate of Young Healthy Smokers," Chest, Vol. 125, No. 1, 2004, pp. 22-26. doi:10.1378/chest.125.1.22

[20] D. Nowak, S. Kalucka, P. Białasiewicz and M. Król, "Exhalation of $\mathrm{H}_{2} \mathrm{O}_{2}$ and Thiobarbituric Acid Reactive Substances (TBARs) by Healthy Subjects," Free Radical Biology \& Medicine, Vol. 30, No. 2, 2001, pp. 178-186. doi:10.1016/S0891-5849(00)00457-3

[21] S. Doruk, H. Ozyurt, H. Inonu, U. Erkorkmaz, O. Saylan and Z. Seyfikli, "Oxidative Status in the Lungs Associated with Tobacco Smoke Exposure," Clinical Chemistry and Laboratory Medicine, 2011, in Press. doi:10.1515/CCLM.2011.698

[22] H. P. Chan, C. R. Lewis and P. S. Thomas, "Oxidative Stress and Exhaled Breath Analysis: A Promising Tool for Detection of Lung Cancer," Cancers, Vol. 2, No. 1, 2010, pp. 32-42. doi:10.3390/cancers2010032

[23] T. P. Szatrowski and C. F. Nathan, "Production of Large Amounts of Hydrogen Peroxide by Human Tumor Cells," Cancer Research, Vol. 51, No. 3, 1991, pp. 794-798.

[24] M. D. Evans, M. Dizdaroglu and M. S. Cooke, "Oxidative DNA Damage and Disease: Induction, Repair and Significance," Mutation Research, Vol. 567, No. 1, 2004, pp. 1-61. doi:10.1016/j.mrrev.2003.11.001
[25] O. Vafa, M. Wade, S. Kern, et al., "c-Myc Can Induce DNA Damage, Increased Reactive Oxygen Species, and Mitigate p53 Function: A Mechanism for Oncogene-Induced Genetic Instability," Molecular Cell, Vol. 9 No. 5, 2002, pp. 1031-1044. doi:10.1016/S1097-2765(02)00520-8

[26] L. Hlavata, H. Aguilaniu, A. Pichova, et al., "The Oncogenic RAS2val19 Mutation Locks Respiration, Independently of PKA, in a Mode Prone to Generate ROS," European Molecular Biology Organization Journal, Vol. 22, No. 13, 2003, pp. 3337-3345.

doi:10.1093/emboj/cdg314

[27] H. Pelicano, D. Carney and P. Huang, "ROS Stress in Cancer Cells and Therapeutic Implications," Drug Resistance Updates, Vol. 7, No. 1, 2004, pp. 97-110. doi:10.1016/j.drup.2004.01.004

[28] D. R. Spitz, E. L. Azzam, J. J. Li and D. Gius, "Metabolic Oxidation/Reduction Reactions and Cellular Responses to Ionizing Radiation: A Unifying Concepts in Stress Response Biology," Cancer and Metastasis Reviews, Vol. 23, No. 1, 2004, pp. 311-322. doi:10.1023/B:CANC.0000031769.14728.bc

[29] J. E. Bigalow, J. B. Mitchell and K. Held, "The Importance of Peroxide and superoxide in the Xray Response," Internal Journal of radiation Oncology, Biology, Physics, Vol. 22, No. 1, 1992, pp. 665-669.

[30] G. R. Buettner, “Ascorbate Oxidation: UV Absorbance of Ascorbate and ESR Spectroscopy of the Ascorbyl Radical as Assay for Iron," Informa Healthcare, Vol. 10, No. 1-2, 1990, pp. 5-9.

[31] L. Boldrini, A. Calcinai, E. Samaritani, et al., "Tumour Necrosis Factor-Alpha and Transforming Growth Factor-Beta Are Significantly Associated with Better Prognosis in Non-Small Cell Lung Carcinoma: Putative Relation with BCL-2-Mediated Neovascularization," British Journal of Cancer, Vol. 83, No. 1, 2000, pp. 480-486. doi:10.1054/bjoc. 2000.1345

[32] L. Gibellini, M. Pinti, M. Nasi, et al., "Interfering with ROS Metabolism in Cancer Cells: The Potential Role of Quercetin," Cancer, Vol. 2, No. 1, 2010, pp. 1288-1311.

[33] Z. Cheng, C. R. Lewis, P. S. Thomas, et al., "Comparative Proteomics Analysis of Exhaled Breath Condensate in Lung Cancer Patients," Journal of Cancer Therapy, Vol. 2, No. 1, 2011, pp. 1-8. doi:10.4236/jct.2011.21001

[34] I. Horvath, J. Hunt and P. J. Barnes, "Exhaled Breath Condensate: Methodological Recommendations and Unresolved Questions," European Respiratory Journal, Vol. 26, No. 3, 2005, pp. 523-548. doi:10.1183/09031936.05.00029705

[35] F. Imer, E. Aldemir, H. Kilic, et al., "The Protective Effect of Amino Acids on the Copper(II)-Catalyzed Autoxidation of Ascorbic Acid," Journal of Food and Drug Analysis, Vol. 16, No. 6, 2008, pp. 46-53.

[36] M. N. Khan and A. Sarwar, "The Influence of Transition Metal Ions on the Kinetics of Ascorbic Acid Oxidation by Methylene Blue in Strongly Acidic Media," Turkish Journal of Chemistry, Vol. 25, No. 1, 2001, pp. 433-440. 


\section{Abbreviations}

EBC: Exhaled breath condensate

ELISA: Enzyme-linked immunosorbent assay

NSCLC: Non small cell lung cancer
ROS: Reactive oxygen species

TNF- $\alpha$ : Tumour Necrosis Factor-alpha

VOCs: Volatile organic compounds 